\title{
Pressure-dependence of Curie temperature and resistivity in complex Heusler alloys
}

\author{
S.K. Bose \\ Department of Physics, Brock University, St. Catharines, Ontario, Canada, L2S $3 A 1$ \\ J. Kudrnovský and V. Drchal \\ Institute of Physics, Academy of Sciences of the Czech Republic, CZ-182 21 Praha 8, Czech Republic \\ I. Turek \\ Charles University, Faculty of Mathematics and Physics, \\ Department of Condensed Matter Physics, Ke Karlovu 5, CZ-12116 Prague 2, Czech Republic
}

(Dated: October 23, 2018)

\begin{abstract}
Using first-principles electronic structure calculations, we have studied the dependence of the Curie temperature on external hydrostatic pressure for random $\mathrm{Ni}_{2} \mathrm{MnSn}$ Heusler alloys doped with $\mathrm{Cu}$ - and $\mathrm{Pd}$-atoms, over the entire range of dopant concentrations. The Curie temperatures are calculated by applying random-phase approximation to the Heisenberg Hamiltonian whose parameters are determined using the linear response and multiple scattering methods, based on densityfunctional theory. In $\left(\mathrm{Ni}_{1-x}, \mathrm{Pd}_{x}\right)_{2} \mathrm{MnSn}$ alloys the Curie temperature is found to increase with applied pressure over the whole concentration range. The crossover from the increase to the decrease of the Curie temperature with pressure takes place for $\mathrm{Cu}$-concentrations larger than about $70 \%$ in $\left(\mathrm{Ni}_{1-x}, \mathrm{Cu}_{x}\right)_{2} \mathrm{MnSn}$ Heusler alloys. The results for the reference $\mathrm{Ni}_{2} \mathrm{MnSn}$ Heusler alloy agree well with a previous theoretical study of Sasioglu et al. and also reasonably well with available experimental data. Results for the spin-disorder induced part of the resistivity in $\left(\mathrm{Ni}_{1-x}, \mathrm{Pd}_{x}\right)_{2} \mathrm{MnSn}$ Heusler alloys, calculated by using the disordered local moment model, are also presented. Finally, a qualitative understanding of the results, based on Anderson's superexchange interaction and Stearn's model of the indirect exchange interaction between localized and itinerant $d$-electrons, is provided.
\end{abstract}

PACS numbers: 71.55.Ak,72.25.Ba,75.10.Hk,75.30.Et

\section{INTRODUCTION}

High pressure studies form an important area of research in solid state physics. Over the years such studies have provided useful insight into our understanding of the

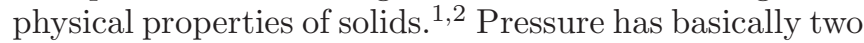
effects on the electrons in solids: increased kinetic energy and accompanying change in the effects of Coulomb interaction among the electrons. In typical band structure calculations the former effect is captured via increased overlap of the basis orbitals, leading to increased hopping matrix elements and band broadening. The changes in the correlation effects are captured via self-consistency of charge and potential, as dictated by the density functional theory and its variants (e.g. generalized gradient approximation (GGA), local density approximation with on-site Coulomb interaction $(\mathrm{LDA}+\mathrm{U}))$. Both of these affect magnetic properties of materials such as exchange interaction, local moments, Curie temperature and resistivity due to magnetic scattering. Of particular importance for metallic magnets are the change in the Fermi surface and the hybridization between different orbitals, which influences the itinerant vs. localized nature of the charge carriers. It is a useful exercise to explore how the existing theories of electronic and magnetic structure calculation fare in describing the pressure variation of these physical properties. In the present paper we study the pressure-dependence of the Curie temperature and the resistivity of some quaternary Heusler alloys, which form an important class of magnetic materials, with potential industrial/technological applications $\underline{\underline{3}} \underline{\underline{3}} \underline{\underline{5}}$ The present work is a natural continuation of our previous paper ${ }^{6}$ in which we presented an extensive study of the magnetic and transport properties of these alloys at ambient pressure.

There exists a number of experimental studies of the variation of the Curie temperature $\left(\mathrm{T}_{c}\right)$ under pressure for elemental ferromagnets, transition-metal alloys,$\frac{7.8}{, 10}$ as well as Heusler alloys $9-11$ including $\mathrm{Co}_{2} \mathrm{TiAl}, \stackrel{10}{\underline{10}}$ $\mathrm{Ni}_{2} \mathrm{MnSn}, \stackrel{9,11}{=} \mathrm{Pd}_{2} \mathrm{MnSn}$ and some others $\stackrel{9}{\underline{9}}$ Recently diluted magnetic semiconductors $\frac{12}{2}$ and some random Heusler alloys 13 have also been studied. However, the corresponding experimental studies of the pressuredependence of the resistivity are rare: one example of such a study is the work by Austin and Mishra $\underline{\underline{9}}$

Several studies of the ambient/equilibrium electronic and magnetic properties of such alloys have appeared recently $\underline{6,14}-18$ Theoretical studies of the pressuredependence of the Curie temperature of elemental transition metal ferromagnets include model-based studies 19,20 as well as recent first-principles calculations. ${ }^{21,22}$ Turek et $a l \stackrel{23}{\underline{h}}$ have studied the pressure-dependence of the Curie temperature in hcp Gd and found a strong dependence of $T_{c}$ on the $c / a$ ratio, which, they suggested, can be related to the measured $T_{c}$ of thick epitaxial $\operatorname{Gd}(0001)$ films on various transition-metal substrates. Similar calculations have also been carried out for the intermetallic 
compound $\mathrm{GdAl}_{2}{ }^{24}$ and for Cr-based compounds in the zinc blende structure 25 A systematic theoretical study of the pressure-dependence of $T_{c}$ in Heusler alloys was carried out recently by Sasioglu et al. $\frac{26}{\underline{2}}$ where the experimentally observed increase of $T_{c}$ with pressure ${ }^{9.11}$ in $\mathrm{Ni}_{2} \mathrm{MnSn}$ was reproduced correctly, albeit only qualitatively so. There exists some other studies of Heusler alloys in which the dependence of the exchange integrals on volume contraction/expansion ${ }^{17.27-29}$ was explored.

The particular Heusler systems considered in this work are the $\left(\mathrm{Ni}_{1-x}, \mathrm{Cu}_{x}\right)_{2} \mathrm{MnSn}$ and $\left(\mathrm{Ni}_{1-x}, \mathrm{Pd}_{x}\right)_{2} \mathrm{MnSn}$ alloys. We employ the coherent potential approximation (CPA) to treat the disorder in the Ni-sublattice due to doping with $\mathrm{Cu}$ - or Pd-atoms. It is well known that CPA neglects the effects of short-range order, which are often present in random alloys. However, for the cases under study, such effects should be weak, as we need to describe the magnetic interactions on the non-random Mn sublattice, which is only indirectly influenced by the randomness of the transition-metal sublattice via hybridization. The most important effect of disorder is, therefore, the change in carrier concentration, which is well-described by the CPA. In a recent paper,$\underline{30}$ it was demonstrated that the CPA provides a reasonably good description of the electronic structure for the case of disorder on the magnetic sublattice as well.

\section{FORMALISM}

The electronic structure calculations are performed employing the tight-binding linear muffin-tin orbital (TB-LMTO) basis 31 and the density functional theory (DFT). The local spin-density approximation (LSDA) for the exchange-correlation part of the potential is used. The effect of substitutional disorder among $(\mathrm{Ni}, \mathrm{Cu})$ or $(\mathrm{Ni}, \mathrm{Pd})$ atoms is described by the coherent potential approximation (CPA) as formulated in the framework of the TB-LMTO Green function method $\stackrel{32}{\underline{3}}$ The calculations employ an $s, p, d, f$-basis, the same atomic radii are adopted for all atoms, and the Vosko-Wilk-Nusair exchange-correlation potential ${ }^{33}$ is used.

The exchange interactions and Curie temperatures are studied here by employing a classical Heisenberg Hamiltonian

$$
H=-\sum_{i \neq j} J_{i j} \mathbf{e}_{i} \cdot \mathbf{e}_{j},
$$

where $J_{i j}$ denotes the exchange integral between Mn atoms at sites $i$ and $j$, and $\mathbf{e}_{i}$ and $\mathbf{e}_{j}$ are unit vectors in the directions of the local magnetization on sites $i$ and $j$, respectively. The parameters of the Heisenberg Hamiltonian are obtained using a two-step approach $\stackrel{34.35}{ }$ The reference state for our calculations is chosen to be the disordered local moment (DLM) state $\underline{\underline{36}}$ which was suc-

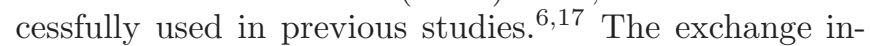
teractions $J_{i j}$ can be expressed in the framework of the
TB-LMTO method as

$$
J_{i j}=\frac{1}{4 \pi} \operatorname{Im} \int_{C} \operatorname{tr}_{L}\left\{\Delta_{i}(z) \bar{g}_{i j}^{\uparrow}(z) \Delta_{j}(z) \bar{g}_{j i}^{\downarrow}(z)\right\} \mathrm{d} z,
$$

where $\Delta_{i}(z)$ characterizes the exchange splitting of the Mn atom at the site $i, \bar{g}_{i j}^{\sigma}(z)$ is the configurationally averaged Green function describing the motion of an electron with spin $\sigma, \sigma=\uparrow, \downarrow$, between Mn sites $i$ and $j$, and the integration is done over the contour $C$ in the complex energy plane $z$ which starts below the valence band and ends at the Fermi energy. Symbol $\operatorname{tr}_{L}$ denotes the trace over the basis orbitals of angular momentum symmetry $L \equiv(\ell, m)$. The effective exchange splitting $\Delta_{i}(z)$ is defined in the TB-LMTO method in terms of the potential functions $P_{i}^{\sigma}(z)$ of Mn atoms as $\Delta_{i}(z)=P_{i}^{\uparrow}(z)-P_{i}^{\downarrow}(z)$. Further details on the exchange interactions evaluated in the DLM reference state can be found in Ref 37 .

We have found that the mapping can be improved (providing a better agreement of calculated $T_{c}$ with the experiment) by including the electron correlation effect on $\mathrm{Ni}$-sites in the framework of the LSDA $+\mathrm{U}$ method (see Fig. 1 in Ref[6]). The electron correlations on Pd-sites are found to have negligible influence on the calculated $T_{c}$. We determine the Curie temperature corresponding to the Heisenberg Hamiltonian using the RPA ${ }^{35}$ and by including all exchange interactions up to $\sim 4$ lattice constants. The pressure is simulated by reduction of the lattice constants from their ambient pressure values, as reported in experiments (see Table 4 of Ref 38 and Table 2 of Ref 39). In Ref 11 an empirical pressure-volume relation was given for $\mathrm{Ni}_{2} \mathrm{MnSn}$. Although such a relation can be obtained entirely from theoretical calculations, in the present study, as in Ref 26, we simply reduce the lattice constant and estimate the corresponding pressure from the empirical pressure-volume relation. According to both the empirical pressure-volume relation 11 and theoretical calculations, $\stackrel{26}{=}$ a $3 \%$ reduction of the lattice constant corresponds roughly to a pressure of $16 \mathrm{GPa}$ in $\mathrm{Ni}_{2} \mathrm{MnSn}$.

In Heusler alloys $\left(\mathrm{Ni}_{1-x}, \mathrm{~T}_{x}\right)_{2} \mathrm{MnSn}(\mathrm{T}=\mathrm{Cu}, \mathrm{Pd})$ the effect of disorder among the $\mathrm{Ni}$ - and $\mathrm{T}$-atoms at the Fermi energy $E_{F}$ is weak because the Ni- and T-states lie well below $E_{F}, \underline{\underline{6}}$ Consequently, the corresponding residual resistivity is small. The resistivity due to phonon scattering $\underline{40}$ is known to be small as well. The dominant contribution to the resistivity, thus, is from the spin-disorder scattering, which increases with temperature, reaching its maximum value at $T_{c}$. In Ref $[6$ we employed a simplified approach to estimate the temperature-dependent resistivity in $\left(\mathrm{Ni}_{1-x}, \mathrm{Pd}_{x}\right)_{2} \mathrm{MnSn}$ Heusler alloys. It was shown that the spin-disorder at and above $T_{c}$ can be described satisfactorily using the DLM model, and the corresponding resistivity can be described by the Kubo-Greenwood approach adapted to the TB-LMTO method. $\underline{41}$ We have used the same approach to study the resistivity under pressure as well. For further details concerning computational techniques we refer the reader to our recent paper $\underline{\underline{6}}$ 
The method we have used is based on mapping the $(T=0)$ total energy to an effective Heisenberg Hamiltonian. In fact it is just the band energy, not the total energy, which is considered in the mapping, by appealing to the so-called 'magnetic force theorem'(see Ref.(25) and references therein). For finite temperature $(T \neq 0)$ calculations, as would be appropriate for the DLM reference state, one needs to consider the mapping of the free energy $F=U-T S$, where $U$ is the internal energy and $S$ is the entropy. $U$ should include the electronic and the average vibrational energy and $S$ should include both electronic and vibrational entropy. Electronic energy should be calculated via finite temperature density functional theory. This free energy cannot, in principle, be mapped to an effective Heisenberg form, which includes only the electronic parameter (spin or magnetic moment). Such a scheme could be implemented for a 'supercell' calculation. However, the advantage of being able to calculate exchange interactions up to a large distance with relative ease and reasonable accuracy and therefore predict results and trends for a group of materials will be gone. For finite temperatures, both electronic excitations and lattice vibrations will influence magnetic properties. However, in the absence of an accurate scheme, we will refrain from speculating on these effects on our results.

\section{RESULTS AND DISCUSSION}

In this section we present results for the pressuredependence of the Curie temperature and spin-disorder resistivity of $\left(\mathrm{Ni}_{1-x}, \mathrm{~T}_{x}\right)_{2} \mathrm{MnSn}(\mathrm{T}=\mathrm{Cu}, \mathrm{Pd})$ Heusler alloys over a broad range of concentrations.

\section{A. Curie temperature under pressure: previous studies}

We start with a brief review of previous theoretical studies, both model-based and first-principles. Theoretical study of the pressure-dependence of $T_{c}$ on a model level has usually been based on a discussion of the indirect exchange interaction among spins 19 or on the Hubbard model of magnetism. ${ }^{20}$ It is clear that the pressuredependence of $T_{c}$ carries information about the volumedependence of the electron wave functions 19 (Bloch functions in the case of crystalline solids). In an RKKYtype indirect exchange interaction model, the volumedependence of $T_{c}$ can be related to the volume dependencies of the density of states at the Fermi level $N\left(E_{F}\right)$ and the exchange integral or the matrix element of the exchange interaction between the wave functions at the Fermi energy $\underline{19}$ (henceforth referred to as the bare exchange interaction $J^{\text {bare }}$, see Eq.(3)). The density of states at the Fermi level usually decreases with pressure (unless, under pressure, new bands start crossing the Fermi level), as the bands broaden due to increased overlap between the orbitals centered on neighboring atoms.
The bare exchange integral usually increases under pressure, due to increased overlap of the wave functions. The above two effects compete with each other in determining the final change of $T_{c}$ under pressure.

In a description based on the Hubbard model, the band terms and the term involving the intra-atomic Coulomb interaction $U$ are written separately. In this case it is possible to divide the volume/pressure-dependence of $T_{c}$ into a term originating from the band broadening under pressure and another that relates to the volume-dependence of $U$. The importance of this latter term for the pressuredependence of $T_{c}$ in $\mathrm{Ni}$ and $\mathrm{Ni}-\mathrm{Cu}$ alloys was first pointed out by Lang and Ehrenreich 20 They argued that it is this term involving the intra-atomic Coulomb interaction that is responsible for the increase in $T_{c}$ under pressure $(P)$ in $\mathrm{Ni}$ and the decrease of $d T_{c} / d P$ with the increase of $\mathrm{Cu}$-concentration in $\mathrm{Ni}-\mathrm{Cu}$ alloys.

In ab initio calculations based on DFT, such as ours, the above effects are merged together and it is hard to delineate one from the other. The advantage, of course, is that the exchange interactions are realistically calculated for the system at hand, and no assumption related to the volume-dependence of any parameter needs to be made. Prototypical studies in this direction are Refs,21,22, addressing the pressure-dependence of the Curie temperature of bcc iron. In particular, a very recent firstprinciples study ${ }^{22}$ seems to agree with experiment, which gives essentially no pressure-dependence of $T_{c}$. The authors describe this result as a balance between the band structure effect which reduces the magnetization and an increase of the bare exchange integrals with decreasing volume, as mentioned above. A somewhat different situation is encountered for fcc-Ni. This metal is a strong ferromagnet, its magnetic moment depends on pressure only weakly and an increase of exchange integrals under pressure thus dominates. As a result, $T_{c}$ of fcc-Ni increases with pressure. In general both effects are relevant and the observed change of $T_{c}$ under pressure is a result of the competition between them.

The above-mentioned competition of the two effects is illustrated in Fig. 1. Here we plot the effective exchange interactions $J_{i j}$ ( Eq22), which control directly the Curie temperature of the system (Fig. 1h), and the bare exchange interactions $J_{i j}^{\text {bare }}$, defined as

$$
J_{i j}^{\text {bare }}=J_{i j} /\left(M_{i} M_{j}\right)
$$

as a function of the distance between the Mn atoms in $\mathrm{Ni}_{2} \mathrm{MnSn}$. We consider the ambient pressure case, as well as elevated pressure simulated by reduction of the lattice constant. In Eq.(3) $M_{i}$ denotes the size of the local magnetic moment of $\mathrm{Mn}$ atom at site $i$ (Fig. 1 b). This definition is motivated by the presence of the exchange splittings $\Delta_{i}(z)$ in Eq. (2) which are roughly proportional to the moment magnitudes $M_{i}$. The increase of the bare exchange interactions with pressure is clearly seen for the first five neighbors (see Fig. 10). It is also obvious that magnetic moments decrease with the pressure, as expected. These two effects, i.e., enhancement of 


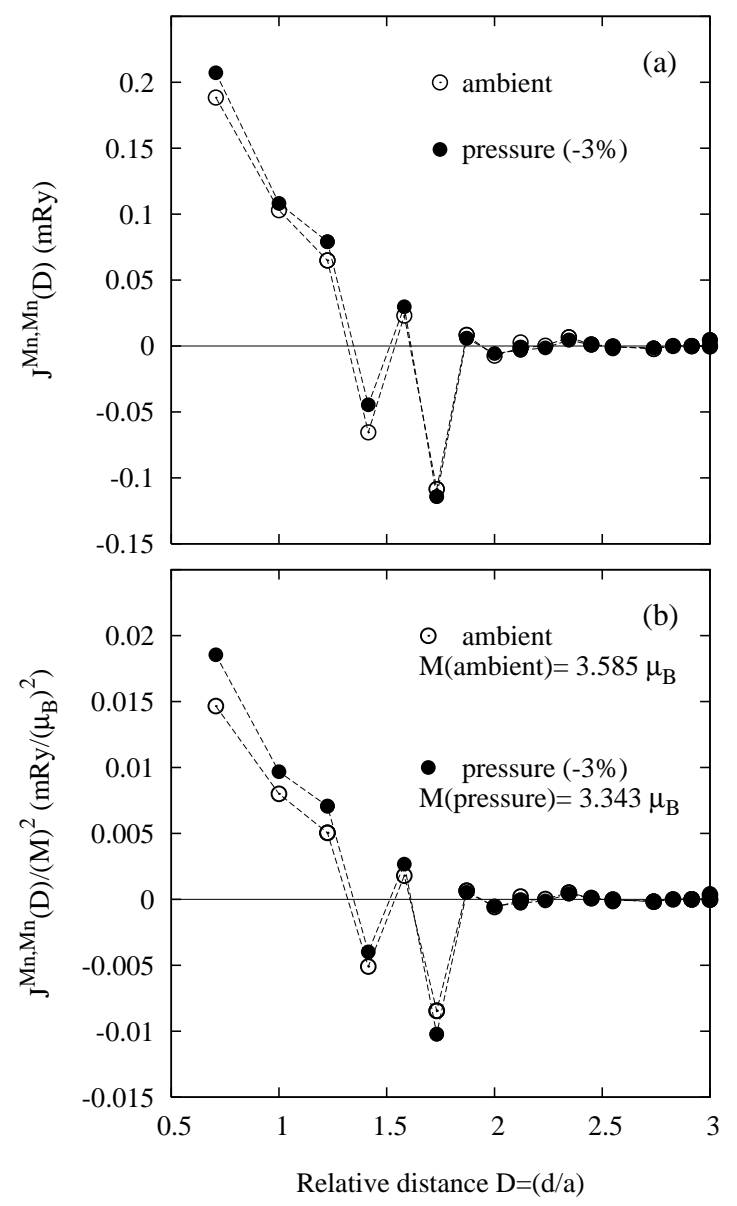

FIG. 1: The Mn-Mn exchange interactions in the $\mathrm{Ni}_{2} \mathrm{MnSn}$ Heusler alloy as functions of the relative interatomic distance $d / a$ where $a$ is the fcc lattice parameter: (a) the effective interactions $J_{i j}$ calculated from Eq. (2), (b) the bare interactions $J_{i j}^{\text {bare }}$ defined by Eq. (3). The cases of ambient pressure (open circles) and the pressure corresponding to the $3 \%$ reduction of the lattice constant (filled circles) are shown.

$J_{i j}^{\text {bare }}$ and suppression of magnetic moments, both due to the volume decrease, compete with each other, resulting in smaller pressure-induced changes of the effective interactions $J_{i j}$ (Fig. 17). The final result in the present case is an enhancement of $T_{c}$ under hydrostatic pressure. The same qualitative explanation was put forward by Csontos et al. ${ }^{12}$ for the increase of $T_{c}$ with pressure in some diluted magnetic semiconductors.

\section{B. Calculated results}

In this section we present and compare results for $(\mathrm{Ni}, \mathrm{Pd})_{2} \mathrm{MnSn}$ and $(\mathrm{Ni}, \mathrm{Cu})_{2} \mathrm{MnSn}$ Heusler alloys, with $\mathrm{Ni}_{2} \mathrm{MnSn}$ considered as the reference case.

\section{1. $\mathrm{Ni}_{2} \mathrm{MnSn}$ and $\mathrm{Pd} \mathrm{d}_{2} \mathrm{MnSn}$ Heusler alloys}

The Heusler alloy $\mathrm{Ni}_{2} \mathrm{MnSn}$ was studied theoretically in great detail in a recent paper, 26 while corresponding experimental results can be found in Refs 9 and 11. The experiments give the rate of increase of $T_{c}$ with pressure around $0.62 \mathrm{~K} / \mathrm{kbar}^{9}$ (for pressures up to $10 \mathrm{kbar}$ or $1 \mathrm{GPa}$ ) and $0.744 \mathrm{~K} / \mathrm{kbar}$ for higher pressures up to $9 \mathrm{GPa} .11$ The authors of the theoretical study ${ }^{26}$ employ the frozen-magnon approach and inverse lattice Fourier transform to construct the effective Heisenberg Hamiltonian, which is then analyzed in the framework of the multisublattice MFA. They obtain an increase of $T_{c}$ by $38 \mathrm{~K}$ for pressures around $16 \mathrm{GPa}$, an increase from the ambient value $T_{c}=362 \mathrm{~K}$ to $T_{c}=400 \mathrm{~K}$. Our estimated values are $T_{c}=322(334) \mathrm{K}$ for ambient pressure and $T_{c}=375$ (401) K (an increase of 53 (67) K for $3 \%$ reduction of the lattice constant, roughly corresponding to the same pressure $\sim 16 \mathrm{GPa})$. The values in brackets correspond to the model assuming electron correlations on Ni-sites (the effective Hubbard $U_{\mathrm{Ni}}$ chosen is $2 \mathrm{eV}$ ). Assuming linear relation between $T_{c}$ and pressure, the experimental increase is expected to be around $100-120 \mathrm{~K}$. The theory thus correctly predicts a pressure-induced increase of $T_{c}$, while the absolute increase is at least three times (Ref 26) to 1.5-1.8-times (present study) too small. The experimental data for $\mathrm{Pd}_{2} \mathrm{MnSn}$ Heusler alloys that we have come across in the literature $\frac{9}{}$ also indicate an increase of $T_{c}$ with pressure at a rate of $0.75 \mathrm{~K} / \mathrm{kbar}$, the same 11 or almost the same ${ }^{9}$ as that for $\mathrm{Ni}_{2} \mathrm{MnSn}$. We have correctly obtained an increase of $T_{c}$ for $3 \%$ reduction of the ambient pressure lattice constant, as in the case of $\mathrm{Ni}_{2} \mathrm{MnSn}$. Specifically, $T_{c}$ is increased by $40 \mathrm{~K}$, a value smaller than but still comparable to that for $\mathrm{Ni}_{2} \mathrm{MnSn}$ and in reasonable agreement with experiment. We find that any additional (i.e., in addition to that given by LSDA) Coulomb term $U$ on $\mathrm{Pd}$-sites has a negligible effect on $T_{c}$ or its pressure-dependence.

The hockey-stick-like variation of the composition dependence of $T_{c}$ for $\left.\mathrm{Ni}_{1-x}, \mathrm{Pd}_{x}\right)_{2} \mathrm{MnSn}$ alloys at ambient pressure is captured in the RPA, but not in the MFA. The RPA is equivalent to including the reaction field (or the Onsager cavity field) effect in the calculation of $T_{c}$ (see, for example, Cyrot $\frac{42}{2}$ and references therein). Our results point to the importance of including the reaction field effect in reproducing the correct (experimentally observed) composition-dependence of $T_{c}$ in this alloy system.

The temperature can influence the above results, as discussed at the end of section II] Increasing temperature leads to an expansion of the lattice, compensating somewhat for the pressure-caused contraction. In essence, thus, the above reasoning should be applied to a net effective reduction of volume, considering both temperature and pressure effects. Lattice expansion effects and the effect of including the Fermi-Dirac distribution function in performing the exchange integrals in Eq. (2) have been discussed recently by Alling et al. $\frac{17}{1}$ However, a complete $T \neq 0$ calculation, as described in section II. 


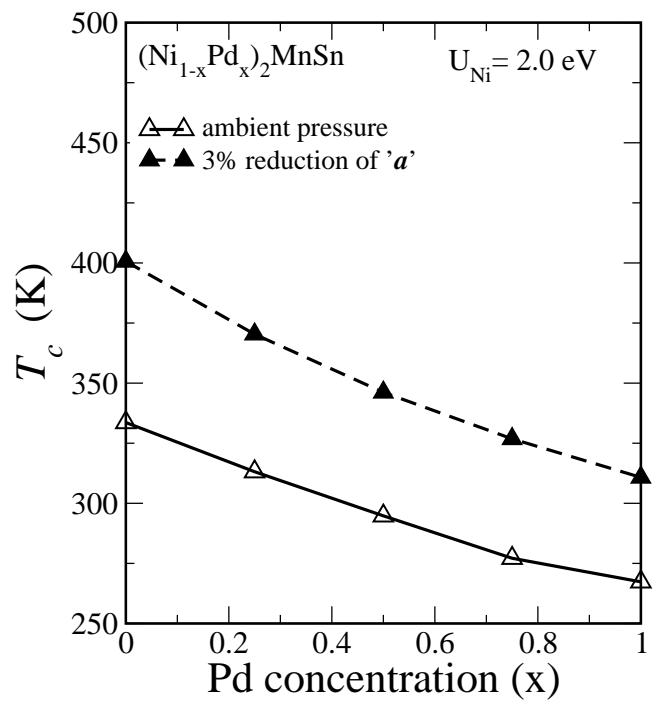

FIG. 2: The concentration-dependence of Curie temperatures (RPA) for $\left(\mathrm{Ni}_{1-x}, \mathrm{Pd}_{x}\right)_{2} \mathrm{MnSn}$ alloys for ambient pressure and for the pressure corresponding to the reduction of the alloy lattice constant by $3 \%$. Model assumes electron correlations on Ni-sites treated in the framework of the LSDA+U method.

has not yet been done.

\section{2. $\left(N i_{1-x}, P d_{x}\right)_{2} M n S n$ Heusler alloys}

The results of the pressure-dependence of $T_{c}$ for $(\mathrm{Ni}, \mathrm{Pd})_{2} \mathrm{MnSn}$ alloys are shown in Fig. 2, We have already mentioned that the ratio by which $T_{c}$ increases with pressure for the end-point alloys is similar for both systems. This is also reflected in the concentrationdependence of $T_{c}$, namely a similar increase of $T_{c}$ under pressure over the whole concentration range. The monotonic and essentially linear concentration-dependence of $T_{c}$ under pressure reflects a similar trend of exchange interactions found for the case of ambient pressure (see $\operatorname{Ref}(6)$. This similarity is due to the isoelectronic nature of Ni- and Pd-atoms coming from the same column, but different rows (3- and 4 - $d$, respectively) of the Periodic Table.

\section{3. $\left(N i_{1-x}, C u_{x}\right)_{2} \mathrm{MnSn}$ Heusler alloys}

Results of a similar study for $(\mathrm{Ni}, \mathrm{Cu})_{2} \mathrm{MnSn}$ alloy over a set of pressures realized by a linear reduction of the alloy lattice constant from the ambient value up to $3 \%$ are shown in Fig. 3. For comparison with experiments, results for the ambient pressure are slightly improved for the present LSDA+U model with respect to the LSDA results of our previous study $\underline{\underline{6}}$ However, both LSDA and $\mathrm{LSDA}+\mathrm{U}$ reveal the same general trend. Specifically, we observe two concentration regions, the first one for $x \leq 0.4$ in which $T_{c}$ is essentially constant with a shallow

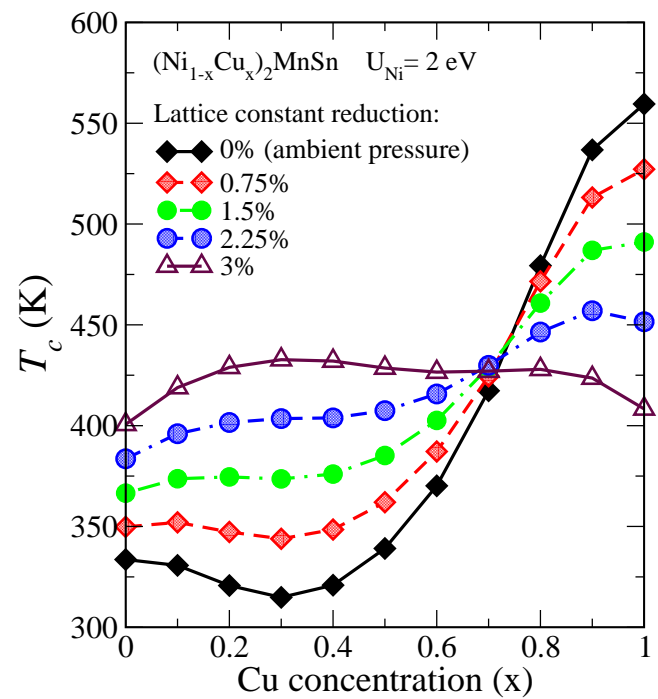

FIG. 3: (Color online) The concentration-dependence of Curie temperatures (RPA) for $\left(\mathrm{Ni}_{1-x}, \mathrm{Cu}_{x}\right)_{2} \mathrm{MnSn}$ alloys for ambient pressure and for set of pressures corresponding to indicated reductions of the alloy lattice constant. Model assumes electron correlations on Ni-sites treated in the framework of the LSDA+U method.

minimum and that for $x>0.4$ where $T_{c}$ increases monotonically with $x$. With increasing pressure the behavior in the first concentration region remains unchanged, but there is a gradual reduction of the slope in the other concentration region (the $\mathrm{Cu}$-rich end). Such a development is clearly related to opposite trends in the pressuredependence of $T_{c}$ in $\mathrm{Ni}_{2} \mathrm{MnSn}$ and $\mathrm{Cu}_{2} \mathrm{MnSn}$, a reduction of $T_{c}$ with pressure for $\mathrm{Cu}_{2} \mathrm{MnSn}$ and an increase for $\mathrm{Ni}_{2} \mathrm{MnSn}$. The crossover from the positive derivative of the pressure-dependence of $T_{c}$ to the negative one takes place around $x=0.7$. Thus, for the pressure corresponding to a $3 \%$ reduction of the lattice constant we predict only a weak dependence of $T_{c}$ on the alloy composition.

\section{Qualitative understanding of results}

In this subsection we will present a qualitative understanding of the composition-dependence of $T_{c}$ at ambient pressure as well as the effect of pressure on $T_{c}$ using the idea of Anderson's superexchange $\frac{27,43}{\text { interaction }}$ and Stearns $\underline{44}$ model of the indirect (RKKY-type) interaction in ferromagnetic Heusler alloys.

The Mn-Mn exchange interaction, as in most other cases, can be divided into three groups: direct, indirect and superexchange. Because the Mn-atoms are not the nearest neighbors of themselves and have a large separation $\left(d_{\mathrm{Mn}-\mathrm{Mn}}>4 \AA\right)$, the direct interaction is not of any importance in Heusler alloys. The most important interaction turns out to be the RKKY-type indirect interaction $\underline{27}$ Of somewhat minor importance is the superexchange interaction, introduced in a series of papers 
by Anderson $\stackrel{43}{ }$ Superexchange, which is also an indirect interaction, is antiferromagnetic, while the RKKYtype interaction can be ferromagnetic or antiferromag-

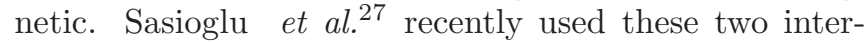
actions to interpret the results for some Heusler alloys. Superexchange is related to possible transitions from occupied states at the Fermi level $E_{F}$ to unoccupied levels above, in the present case to the unoccupied minority Mn-bands. Because the superexchange interaction is negative, the stronger the superexchange part, the smaller is the total exchange integral (and $T_{c}$ ). The strength of the superexchange interaction depends on the density of states (DOS) at $E_{F}$ and the energy separation between $E_{F}$ and the unoccupied Mn-bands. The larger the DOS at $E_{F}$ and/or the smaller the above energy separation, the stronger is the superexchange. This lends a qualitative understanding of the increase of $T_{c}$ from $\mathrm{Pd}_{2} \mathrm{MnSn}$ to $\mathrm{Ni}_{2} \mathrm{MnSn}$ to $\mathrm{Cu}_{2} \mathrm{MnSn}$, if the DOS mechanism dominates over the decreasing of energy separation in the above sequence of alloys (Fig.2 of Ref, 6 ). If we dope the $\mathrm{Ni}$-sublattices with $\mathrm{Cu}$-atoms in the $\mathrm{Ni}_{2} \mathrm{MnSn}$ host, the observed hockey-stick-like composition-dependence of $T_{c}$ can be understood as a competition of above two effects: for low $\mathrm{Cu}$-content the decrease of $T_{c}$ with increasing $\mathrm{Cu}$-concentration is due to reduction of the above energy separation, causing an increase of the superexchange part of the interaction. For $x_{\mathrm{Cu}}>0.35$ the DOS-effect starts to dominate, with decreasing DOS at $E_{F}$ and thus smaller superexchange and larger total exchange interaction. This leads to higher $T_{c}$ of $\mathrm{Cu}_{2} \mathrm{MnSn}$ as compared to $\mathrm{Ni}_{2} \mathrm{MnSn}$.

In $(\mathrm{Ni}, \mathrm{Pd})_{2} \mathrm{MnSn}$ alloy the DOS-effect is the only important effect over the entire concentration range. Because the $\mathrm{Ni}$ - and $\mathrm{Pd}$-atoms have the same valency, the above mentioned energy separation does not change with concentration. There is a small decrease in the DOS at $E_{F}$ in $\mathrm{Pd}_{2} \mathrm{MnSn}$ compared with $\mathrm{Ni}_{2} \mathrm{MnSn}$ (Fig.2 of Ref $\left[\right.$ ), decreasing the superexchange and increasing $T_{c}$.

As mentioned above, although the superexchange mechanism seems to support the observed results, it cannot be the driving mechanism for the Heusler alloys under study. This is because of relatively large separation of the unoccupied minority Mn-peak above $E_{F}$ (the Mn-DOS just above $E_{F}$ is small). Of much-larger importance is the indirect interaction and its key feature in ferromagnetic Heusler alloys was captured and successfully described by Stearns 44 in a series of papers in the late seventies. Stearns asserts that the magnetism of Heusler alloys (as well as bcc Fe $\underline{45}$ ) can be understood by dividing the $d$ electrons into localized $d$-electrons $\left(d_{l}\right)$ associated with narrow $d$-subbands and itinerant $d$-electrons $\left(d_{i}\right)$ associated with (usually) one broader $d$-subband. The interaction between these localized and itinerant $d$-electrons is similar in nature to RKKY interaction, originally formulated for the interaction between localized moments in a free electron gas. The $d$-band gets narrower across (with increasing number of electrons) a given series and broader with increasing row number (e.g. from $3 d$ to 4d). Thus Stearns used her model to describe correctly the change of $T_{c}$ from $\mathrm{Ni}_{2} \mathrm{MnSn}$ to $\mathrm{Cu}_{2} \mathrm{MnSn}$ (an increase in $d_{l}$ causing a rise of $T_{c}$ ) and from $\mathrm{Ni}_{2} \mathrm{MnSn}$ to $\mathrm{Pd}_{2} \mathrm{MnSn}$ (a decrease of $T_{c}$ due to decreasing $d_{l}$ ). Uhl ${ }^{38}$ used Stearns model to explain the hockey-stick appearance of the composition-dependence of $T_{c}$ with increasing $x$ in $\left(\mathrm{Ni}_{1-x}, \mathrm{Cu}_{x}\right)_{2} \mathrm{MnSn}$ Heusler alloys. With increasing $\mathrm{Cu}$-concentration, the lattice dilates. Initially, this dilation causes a reduction in the strength of the $d_{i}$ - $d_{l}$ interaction, associated with increased $\mathrm{Mn}-\mathrm{Mn}$ distance. However, the addition of $\mathrm{Cu}$ also causes an increase in the number of $d_{l}$ and thus in the $d_{i}-d_{l}$ interaction. The resulting $T_{c}$ thus shows an initial decrease followed subsequently by an increase (for further details, see Uhl ${ }^{38}$ ). Thus both superexchange and indirect $d_{i}-d_{l}$ exchange interaction give rise to the same trend for the compositiondependence of $T_{c}$.

The pressure effect on $T_{c}$ can be understood in the light of the above explanation for the composition-dependence at ambient pressure. Pressure reduces the lattice parameter, an effect opposite to the dilation caused by $\mathrm{Cu}$ addition. Pressure also broadens bands, reducing $d_{l}$ and increasing $d_{i}$, again opposite to what happens with increasing $\mathrm{Cu}$-content. Thus on the Ni-rich side increased pressure increases $T_{c}$ (opposite to the result with $\mathrm{Cu}$ addition), and beyond a certain critical $\mathrm{Cu}$-concentration increasing pressure results in decreasing $T_{c}$ (opposite to the result with $\mathrm{Cu}$-addition).

Similar reasoning explains the compositiondependence of ambient pressure $T_{c}$ and the pressuredependence of $T_{c}$ in $\left(\mathrm{Ni}_{1-x}, \mathrm{Pd}_{x}\right)_{2} \mathrm{MnSn}$ alloys. Doping Ni-sublattice with $\mathrm{Pd}$-atoms results in dilation of the lattice. In addition, d-electrons in $\mathrm{Pd}$ are more delocalized than in Ni. Both of these will lead to a decrease of $T_{c}$ with increasing $\mathrm{Pd}$ concentration. Pressure, via contraction of the lattice, reverses this effect. It appears that in this case the increasing strength of the interaction due to reduction in the $\mathrm{Mn}-\mathrm{Mn}$ distance supersedes the effect of the pressure-induced delocalization of the electrons.

A couple of comments are in order. Stearns model is applicable to only ferromagnetic Heusler alloys. In several semi-Heusler alloys, or in general in situations where the superexchange part of the interaction becomes important, Stearns model may fail to predict the results correctly. It would not be incorrect to point out that without an accurate first-principles study as presented here, it would be almost impossible to capture theoretically the details of the composition- or pressure-dependence of $T_{c}$, although some qualitative understanding (especially, a posteriori) is possible. In this sense the importance of first-principles studies like the present one is irrefutable.

\section{Spin-disorder induced resistivity}

In magnetic alloys there are three contributions to the resistivity: a temperature-independent residual re- 


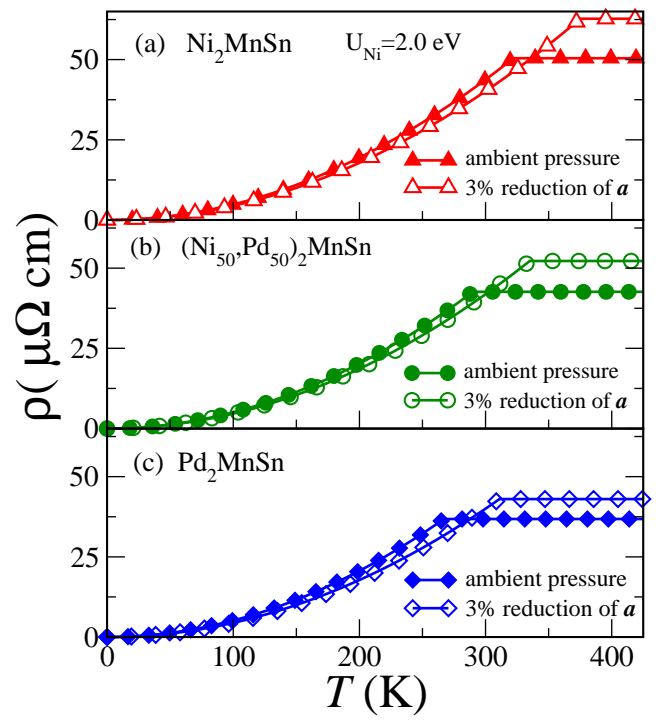

FIG. 4: (Color online) The temperature-dependence of the resistivity of the $\left(\mathrm{Ni}_{1-x}, \mathrm{Pd}_{x}\right)_{2} \mathrm{MnSn}$ alloys for ambient pressure (full symbols) and the pressure corresponding to the reduction of the alloy lattice constant by $3 \%$ (open symbols): (a) $x=0$, (b) $x=0.5$, (c) $x=1$.

sistivity due to the alloy disorder and other defects, and two temperature-dependent contributions, one due to electron-phonon and the other due to electron-magnon scatterings. In the present case, the residual resistivity is small because the states corresponding to $\mathrm{Ni}-, \mathrm{Cu}-$, and Pd-atoms are well below the alloy Fermi energy $\underline{\underline{6}}$ and thus influence states at the Fermi energy (relevant for residual resistivity) only weakly. The contribution due to phonons is well understood, and for Heusler alloys around room temperature,$\underline{40}$ this contribution is known to be small. The dominant contribution is thus due to spin-disorder $\stackrel{46.47}{ }$ The spin-disorder induced resistivity is controlled by the spin-spin correlation function which is small at and above $T_{c}$, so that a reasonable representation of the situation is given by the DLM state, as explained in detail elsewhere $\underline{6}^{-}$Kasuya's $s$ - $d$ interaction model $l^{47}$ shows that the temperature-dependence of spin-disorder resistivity is primarily quadratic and can be well-simulated by the $B T^{2}$-law. In the present case we determine the constant $B$ from first principles using $B=\rho\left(T_{c}\right) / T_{c}^{2}$, where $\rho\left(T_{c}\right)$ is identified with the resistivity of the DLM state. The DLM state is described in the framework of the CPA as an equiconcentration alloy with two types of Mn atoms (A and B) with their moments pointing randomly up or down (collinear disorder). The corresponding resistivity can be properly calculated by the Kubo-Greenwood formula applied to this binary-Mn alloy. The results for $\mathrm{Ni}_{2} \mathrm{MnSn},\left(\mathrm{Ni}_{0.5}, \mathrm{Pd}_{0.5}\right) \mathrm{MnSn}$, and $\mathrm{Pd}_{2} \mathrm{MnSn}$ alloys are shown in Fig. 4. It should be noted that the residual resistivity of the $\left(\mathrm{Ni}_{0.5}, \mathrm{Pd}_{0.5}\right) \mathrm{MnSn}$ alloy in the ferromagnetic state is about two orders of magnitude smaller than that in the DLM state $\underline{\underline{6}}$

The spin-disorder part of the resistivity is controlled by the constant $B$. For the cases shown in Fig. $4, T_{c}$ increases under pressure. Due to band-broadening under pressure and accompanying delocalization of the electrons, $\rho(T)$ decreases in general for a given $T$ less than the ambient pressure $T_{c}$. However, $\rho\left(T_{c}\right)$ (as well as $\rho(T)$ for $T \geq$ ambient pressure $T_{c}$ ) is higher under pressure because of the increase in $T_{c}$.

To our knowledge, there are no available experimental results for the systems studied in this paper, although the results of Austin and Mishra ${ }^{9}$ for related $\mathrm{Pd}_{2} \mathrm{MnSb}$ Heusler alloy do indicate, as in this work as well, an increase of the resistivity under pressure for temperatures above $T_{c}$.

Undoubtedly pressure has non-negligible influence on the part of the resistivity due to phonons. This, however, is beyond the scope of the present article.

\section{CONCLUSIONS}

We have studied magnetic and transport properties of quaternary Heusler alloys $(\mathrm{Ni}, \mathrm{T})_{2} \mathrm{MnSn}(\mathrm{T}=\mathrm{Cu}, \mathrm{Pd})$ under pressure by means of the first-principles density functional method. In particular, we have investigated in detail the pressure-dependence of the Curie temperature. In agreement with experiments, we obtain an increase of the Curie temperature under pressure (reduction of the lattice constant) for $\mathrm{Ni}_{2} \mathrm{MnSn}$ and $\mathrm{Pd}_{2} \mathrm{MnSn}$. On the other hand, $\mathrm{Cu}_{2} \mathrm{MnSn}$ alloy exhibits a reduction of the Curie temperature with applied pressure. The results can be qualitatively understood as an interplay of two effects: an increase of the bare exchange integrals with the volume reduction and the decrease of magnetic moments with pressure due to band broadening.

The concentration-dependence of the Curie temperature under pressure is simple in $(\mathrm{Ni}, \mathrm{Pd})_{2} \mathrm{MnSn}$ Heusler alloys where the increase of the Curie temperature has an almost constant slope. On the other hand, we predict a dramatic change of the Curie temperature with pressure from the positive for Ni-rich $(\mathrm{Ni}, \mathrm{Pd})_{2} \mathrm{MnSn}$ alloys to a negative one in $\mathrm{Cu}$-rich alloys, the crossover being around $70 \%$ of $\mathrm{Cu}$. We emphasize that such a complex behavior cannot be obtained without an accurate calculation of the exchange interaction and a proper statistical treatment to compute $T_{c}$ (i.e., by going beyond the MFA).

A simple explanation of the behavior of the calculated $T_{c}$ under pressure is given in the framework of Anderson's superexchange interaction and the Stearns model of the indirect exchange interaction between itinerant and localized $d$-electrons.

We have also investigated the pressure-dependence of the spin-disorder related part of the resistivity, which dominates at higher temperatures, the residual resistivity due to alloy disorder being small and important only for very low temperatures. We have found that the resistivity at the Curie temperature increases with pressure, but this result is due to the increase of the Curie temperature 
with pressure itself. For temperatures below the ambient pressure $T_{c}$ the resistivity decreases under pressure, as would be expected due to band broadening.

\section{Acknowledgments}

This work was supported by a grant from the Natural Sciences and Engineering Research Council of
Canada. J.K. and V.D. acknowledge financial support from AV0Z 10100520 and the Czech Science Foundation (202/09/0775). The work of I.T. has been supported by the Ministry of Education of the Czech Republic (Grant No. MSM 0021620834) and by the Czech Science Foundation (Grant No. 202/09/0030).
${ }^{1}$ G. Beggerow in Landolt-Börnstein, New Series, Group IV, Vol. 4 High-Pressure Properties of Matter, ed. K. Schäfer (Springer, Berlin, 1980).

2 P.F. McMillan, Nature Materials 1, 19 (2002).

3 P. J. Webster and K. R. A. Ziebeck, "Heusler Alloys," in Magnetic Properties of Metals, Landolt-Börnstein New Series Group III, Vol. 19C, H. R. J. Wijn (Ed.) (Springer, Berlin, 1988) p. 75.

${ }^{4}$ O. Tegus, E. Brück, L. Zhang, Dagula, K.H. J. Buschow, F.R. de Boer, Physica B 319, 174 (2002).

5 T. Krenke, E. Duman, M. Acet, E.F. Wassermann, X. Moya, L. Mañosa, and A. Planes, Nature Materials 4, 450 (2005).

6 S.K. Bose, J. Kudrnovský, V. Drchal, and I. Turek, Phys. Rev. B 82, 174402 (2010).

7 D. Bloch and R. Pauthenet, J. Appl. Phys. 36, 1229 (1965).

8 Y. Yamamoto, N. Nakagiri, M. Nomura, H. Tange, and H. Fujiwara, Jap. J. Appl. Phys. 18, 2139 (1979),

9 I.G. Austin and P.K. Mishra, Philos. Mag. 15, 529 (1967).

10 E. DiMasi, M.C. Aronson, and B.R. Coles, Phys. Rev. B 47, 14301 (1993).

11 A.G. Gavriliuk, G.N. Stepanov, V.A. Sidorov, and S.M. Irkaev, J. Appl. Phys. 79, 2609 (1999).

12 M. Csontos, G. Mihály, B. Jankó, T. Wojtowicz, X. Liu, and J.K. Furdyna, Nature Materials 4, 447 (2005).

13 Y. Chieda et al. , J. Alloys and Compounds 486, 51 (2009).

14 J. Kudrnovský, V. Drchal, and P. Bruno, Phys. Rev. B 77, 224422 (2008).

15 L. Bergqvist, O. Eriksson, J. Kudrnovský, V. Drchal, P. Korzhavyi, and I. Turek, Phys. Rev. Lett. 93, 137202 (2004).

16 J. Kudrnovský, V. Drchal, I. Turek, and P. Weinberger, Phys. Rev. B 78, 054441 (2008).

17 B. Alling, A.V. Ruban, and I.A. Abrikosov, Phys. Rev. B 79, 134417 (2009)

18 J. Rusz, L. Bergqvist, J. Kudrnovský, and I. Turek, Phys. Rev. B 73, 214412 (2006).

19 S.H. Liu, Phys. Rev. 127, 1889 (1962).

20 N.D. Lang and H. Ehrenreich, J. Appl. Phys. 38, 1316 (1967).

21 S. Morán, C. Ederer, and M. Fähnle, Phys. Rev. B 67, 012407 (2003).

${ }^{22}$ F. Körmann, A. Dick, T. Hickel, and J. Neugebauer, Phys. Rev. B 79, 184406 (2009).

${ }^{23}$ I. Turek, J. Kudrnovský, G. Bihlmayer and S. Blügel, J.
Phys.: Condens. Matter15, 2771 (2003).

24 I. Turek, J. Rusz, and M. Diviš, J. Alloys and Compounds 431, 37 (2007).

25 S.K. Bose and J. Kudrnovský, Phys. Rev. B 81, 054446 (2010).

26 E. Sasioglu, L. Sandratskii, and P. Bruno, Phys. Rev. B 71, 214412 (2005).

27 E. Sasioglu, L. Sandratskii, and P. Bruno, Phys. Rev. B 77, 064417 (2008).

28 Y. Kurtulus, R. Dronskowski, G.D. Samolyuk, and V.P. Antropov, Phys. Rev. B 71, 014425 (2005).

29 M. Meinert, J.M. Smalhorst, and G. Reiss, J. Phys.: Condens. Matter 23, 036001 (2011).

${ }^{30}$ K. Ozdogan, E. Sasioglu, and I. Galanakis, J. Appl. Phys. 103, 023503 (2008).

31 O.K. Andersen and O. Jepsen, Phys. Rev. Lett. 53, 2571 (1984).

32 I. Turek, V. Drchal, J. Kudrnovský, M. Šob, and P. Weinberger, Electronic Structure of Disordered Alloys, Surfaces and Interfaces (Kluwer, Boston, 1997).

33 S.H. Vosko, L. Wilk, and M. Nusair, Can. J. Phys. 58, 1200 (1980).

34 A.I. Liechtenstein, M.I. Katsnelson, V.P. Antropov, and V.A. Gubanov, J. Magn. Magn. Mater. 67, 65 (1987).

35 I. Turek, J. Kudrnovský, V. Drchal, and P. Bruno, Philos. Mag. 86, 1713 (2006).

36 B.L. Gyorffy, A.J. Pindor, J. Staunton, G.M. Stocks, and H. Winter, J. Phys. F: Metal Phys. 15, 1337 (1985).

37 M. Ondráček, O. Bengone, J. Kudrnovský, V. Drchal, F. Máca, and I. Turek, Phys. Rev. B 81, 064410 (2010).

38 E. Uhl, Monatshefte für Chemie 113, 275 (1982).

39 E. Uhl, Solid State Commun. 53, 395 (1985).

40 W.H. Schreiner, D.E. Brandao, F. Ogiba, and J.V. Kunzler, J. Phys. Chem. Solids 43, 777 (1982).

41 I. Turek, J. Kudrnovský, V. Drchal, L. Szunyogh, and P. Weinberger, Phys. Rev. B 65, 125101 (2002).

42 M. Cyrot, J. Mag. Mag. Mater. 45, 9 (1984).

43 P.W. Anderson, Phys. Rev. 124, 41 (1961).

44 M.B. Stearns, J. Appl. Phys. 50, 2060 (1979); see also J.R. Reitz and M.B. Stearns, ibid., 2066; M.B. Stearns, J. Mag. Mag. Mater. 15-18, 301 (1980).

45 M.B. Stearns, Phys. Today, April 1978 issue, pp. 34-39.

46 T. Kasuya, Prog. Theor. Phys. 16, 58 (1956).

47 T. Kasuya, Prog. Theor. Phys. 22, 227 (1959). 